\title{
CONSTANTS AND CONVERSION FACTORS
}

Proton mass, $m_{p}=1.67 \times 10^{-27} \mathrm{~kg}$

Neutron mass, $m_{n}=1.67 \times 10^{-27} \mathrm{~kg}$

Electron mass, $m_{e}=9.11 \times 10^{-31} \mathrm{~kg}$

Speed of light, $\quad c=3.00 \times 10^{8} \mathrm{~m} / \mathrm{s}$
Electron charge magnitude, $\quad e=1.60 \times 10^{-19} \mathrm{C}$

Coulomb's law constant, $\quad k=1 / 4 \pi \varepsilon_{0}=9.0 \times 10^{9} \mathrm{~N} \cdot \mathrm{m}^{2} / \mathrm{C}^{2}$

Universal gravitational

constant,

$G=6.67 \times 10^{-11} \mathrm{~m}^{3} / \mathrm{kg} \cdot \mathrm{s}^{2}$

Acceleration due to gravity

at Earth's surface, $g=9.8 \mathrm{~m} / \mathrm{s}^{2}$

\begin{tabular}{|c|c|c|c|c|c|c|c|}
\hline \multirow{4}{*}{$\begin{array}{c}\text { UNIT } \\
\text { SYMBOLS }\end{array}$} & meter, & $\mathrm{m}$ & kelvin, & $\mathrm{K}$ & watt, & W & degree Celsius, \\
\hline & kilogram & $\mathrm{kg}$ & hertz, & $\mathrm{Hz}$ & coulomb, & $\mathrm{C}$ & \\
\hline & second, & $\mathrm{S}$ & newton & $\mathrm{N}$ & volt, & $\mathrm{V}$ & \\
\hline & ampere, & A & joule, & $\mathrm{J}$ & ohm, & $\Omega$ & \\
\hline
\end{tabular}

\begin{tabular}{|c|c|c|}
\hline \multicolumn{3}{|c|}{ PREFIXES } \\
\hline Factor & Prefix & Symbol \\
\hline $10^{12}$ & tera & $\mathrm{T}$ \\
\hline $10^{9}$ & giga & $\mathrm{G}$ \\
\hline $10^{6}$ & mega & $\mathrm{M}$ \\
\hline $10^{3}$ & kilo & $\mathrm{k}$ \\
\hline $10^{-2}$ & centi & $\mathrm{c}$ \\
\hline $10^{-3}$ & milli & $\mathrm{m}$ \\
\hline $10^{-6}$ & micro & $\mu$ \\
\hline $10^{-9}$ & nano & $\mathrm{n}$ \\
\hline $10^{-12}$ & pico & $\mathrm{p}$ \\
\hline
\end{tabular}

\begin{tabular}{|c|c|c|c|c|c|c|c|}
\hline \multicolumn{1}{|c|}{ VALUES OF TRIGONOMETRIC FUNCTIONS FOR COMMON ANGLES } \\
\hline$\theta$ & $0^{\circ}$ & $30^{\circ}$ & $37^{\circ}$ & $45^{\circ}$ & $53^{\circ}$ & $60^{\circ}$ & $90^{\circ}$ \\
\hline $\sin \theta$ & 0 & $1 / 2$ & $3 / 5$ & $\sqrt{2} / 2$ & $4 / 5$ & $\sqrt{3} / 2$ & 1 \\
\hline $\cos \theta$ & 1 & $\sqrt{3} / 2$ & $4 / 5$ & $\sqrt{2} / 2$ & $3 / 5$ & $1 / 2$ & 0 \\
\hline $\tan \theta$ & 0 & $\sqrt{3} / 3$ & $3 / 4$ & 1 & $4 / 3$ & $\sqrt{3}$ & $\infty$ \\
\hline
\end{tabular}

The following conventions are used in this exam.

I. The frame of reference of any problem is assumed to be inertial unless otherwise stated.

II. Assume air resistance is negligible unless otherwise stated.

III. In all situations, positive work is defined as work done on a system.

IV. The direction of current is conventional current: the direction in which positive charge would drift.

V. Assume all batteries and meters are ideal unless otherwise stated. 


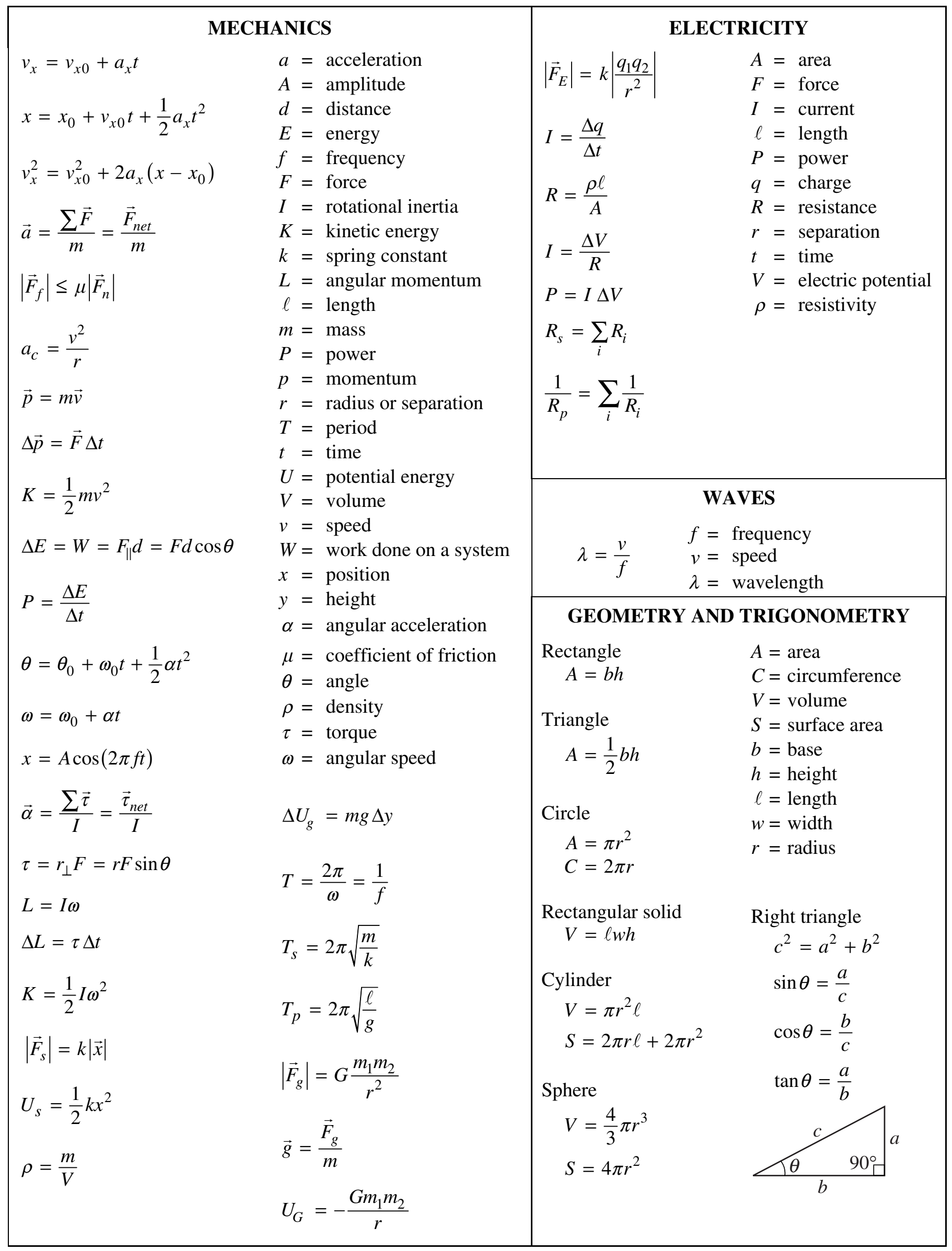

